\title{
Optimization in Transition between Two Dynamic Systems Governed by a Class of Weakly Singular Integro-Differential Equations
}

\author{
Shihchung Chiang \\ Department of Finance, Chung Hua University, Taiwan \\ Email: Chiang@chu.edu.tw
}

How to cite this paper: Chiang, S. (2019) Optimization in Transition between Two Dynamic Systems Governed by a Class of Weakly Singular Integro-Differential Equations. Applied Mathematics, 10, 826-835. https://doi.org/10.4236/am.2019.1010059

Received: September 2, 2019

Accepted: October 7, 2019

Published: October 10, 2019

Copyright $\odot 2019$ by author(s) and Scientific Research Publishing Inc. This work is licensed under the Creative Commons Attribution International License (CC BY 4.0).

http://creativecommons.org/licenses/by/4.0/

\section{(c) (i) Open Access}

\begin{abstract}
This study presents numerical methods for solving the minimum energies that satisfy typical optimal requirements in the transition between two dynamic systems where each system is governed by a different kind of weakly singular integro-differential equation. The class of weakly singular integro-differential equations originates from mathematical models in aeroelasticity. The proposed numerical methods are based on earlier reported approximation schemes for the equations of the first kind and the second kind. The main result of this study is the development of numerical techniques for determining the stability between two dynamic systems in the minimum energy sense.
\end{abstract}

\section{Keywords}

Optimal Requirement, Transition, Weakly Singular Integro-Differential Equations, Stability

\section{Introduction}

The minimum energy problem and the associated optimal control problem have been investigated for more than half a century. The system constraints can be ordinary differential equations, partial differential equations, or functional differential equations. This study introduces a numerical method for finding the minimum energy to satisfy the general criterion that can be adjusted to minimize various requirements through the selection of appropriate parameters. One system constraint is the class of equations of the first kind, which originates from an aeroelasticity problem where the mathematical model consists of eight integro-differential equations [1]. In the model, the most determinate equation 
is a scalar weakly singular integro-differential equation of the first kind [2] [3]. Furthermore, because of the natural facts of transition between liquid water and solid ice [4] or the aviation transition between vertical take-off and horizontal flight of an unmanned aerial vehicle [5], we were interested in the energy issue in the transition between two basically different (but related) dynamic systems. For the setting, the second dynamic system was constructed from the first system using finite derivative delay terms that included the boundary points of the considered interval. This study followed the structure of other relevant studies [6] in assuming that the forcing terms of the system are the control forces. This study is organized as follows: Section 2 presents the criteria for the optimal issues. Section 3 presents the approach for determining the minimum energy for the transition procedure. Section 4 presents the numerical results attained by choosing different parameters for various cost requirements. Section 5 presents the summary of this study.

\section{The Model}

Consider the class of weakly singular integro-differential equations of the first kind

$$
\frac{\mathrm{d}}{\mathrm{d} t} D x_{t}=u(t)
$$

with initial data

$$
x(s)=\phi(s),-b \leq s \leq 0 .
$$

The difference operator $D$ is defined as

$$
D x_{t}=\int_{-b}^{0} g(s) x_{t}(s) \mathrm{d} s,
$$

where

$$
x_{t}(s)=x(t+s) .
$$

The weighting kernel $g$ is integrable, positive, nondecreasing, and weakly singular at $s=0$. The control force $u(t)$ is assumed to be locally integrable for $t>0$. Although a more general kernel $g$ also works, this study focused on the Abel-type kernel (i.e., $g(s)=|s|^{-p}$, where $s \in[-b, 0]$ and $p=0.5$ from the original aeroelastic model).

The initial condition $\phi(s),-b \leq s \leq 0$ is in $L_{1, g}$, which is a weighted $L_{1}$ space with weight $g(\cdot)$. Note that the initial value problem in Equations (1)-(2) can be written as

$$
D x_{t}=D x_{0}+\int_{0}^{t} u(\tau) \mathrm{d} \tau
$$

provided that the function

$$
D x_{t}=\int_{-b}^{0} g(s) x(t+s) \mathrm{d} s
$$

is absolutely continuous for $t>0$ and the function $g(\cdot) \phi(\cdot)$ belongs to $L_{1}[-b, 0]$. Without a loss of generality, we assume that $b=1$. 
The second system is a class of weakly singular integro-differential equations of the second kind

$$
\sum_{i=1}^{l} \frac{\mathrm{d}}{\mathrm{d} t} x\left(t-\sigma_{i}\right)+\frac{\mathrm{d}}{\mathrm{d} t} D x_{t}=u(t)
$$

where $l$ is a positive integer and $0 \leq \sigma_{i} \leq 1, i=1, \cdots, l$. The initial condition is

$$
x(s)=\phi(s),-1 \leq s \leq 0 .
$$

For the partition between systems (2) and (3), a parameter $\lambda \in[0,1]$ is assumed. Therefore, the combined system can be written as

$$
\left\{\begin{array}{l}
\sum_{i=1}^{l} \frac{\mathrm{d}}{\mathrm{d} t} \lambda x\left(t-\sigma_{i}\right)+\frac{\mathrm{d}}{\mathrm{d} t} D \lambda x_{t}=u(t) \\
\frac{\mathrm{d}}{\mathrm{d} t} D(1-\lambda) x_{t}=v(t)
\end{array}\right.
$$

with initial data

$$
x(s)=\phi(s),-1 \leq s \leq 0 .
$$

Although the proposed methods can be applied to more general cost functions, this study primarily considered the typical cost function for comparison:

$$
\Phi(\lambda)=\Phi_{1}(\lambda)+\Phi_{2}(\lambda)
$$

and

$$
\begin{gathered}
\Phi_{1}(\lambda)=\alpha_{1}(\lambda x(1)-h)^{2}+\alpha_{2} \int_{0}^{1}(\lambda x(t)-\eta(t))^{2} \mathrm{~d} t+\alpha_{3} \int_{0}^{1} u(t)^{2} \mathrm{~d} t \\
\Phi_{2}(\lambda)=\alpha_{1}((1-\lambda) x(1)-h)^{2}+\alpha_{2} \int_{0}^{1}((1-\lambda) x(t)-\eta(t))^{2} \mathrm{~d} t+\alpha_{3} \int_{0}^{1} v(t)^{2} \mathrm{~d} t,
\end{gathered}
$$

where $h$ is a constant of final target state, $\eta(t)$ is a target function, and parameters $\alpha_{1}, \alpha_{2}$ and $\alpha_{3}$ are nonnegative constants with a total sum of 1 .

\section{The Numerical Method}

This procedure is proposed to discretize system (9) and the cost function (10) simultaneously to construct two corresponding linear systems with unknowns as states and controls. The space mesh points (corresponding to the $s$ variable) are discretized as $-1=\tau_{n}<\tau_{n-1}<\cdots<\tau_{1}<\tau_{0}=0$, and a new variable $\xi$ is defined as

$$
\xi(t, s)=x(t+s),-1 \leq s \leq 0, t>0 .
$$

System (9) can then be reformulated as a first-order hyperbolic equation

$$
\frac{\partial}{\partial t} \xi(t, s)=\frac{\partial}{\partial s} \xi(t, s),-1 \leq s \leq 0,
$$

with the condition

$$
\left\{\begin{array}{l}
\lambda \sum_{i=1}^{l} \frac{\mathrm{d}}{\mathrm{d} t} \xi\left(t,-\sigma_{i}\right)+\lambda \int_{-1}^{0}|s|^{-p} \frac{\partial}{\partial s} \xi(t, s) \mathrm{d} s=u(t), \\
(1-\lambda) \int_{-1}^{0}|s|^{-p} \frac{\partial}{\partial s} \xi(t, s) \mathrm{d} s=v(t) .
\end{array}\right.
$$


Next, assume that the solution to Equation (8) has the form

$$
\xi(t, s)=\sum_{i=0}^{n} \kappa_{i}(t) B_{i}(s)
$$

where the basis, $B_{i}(s), i=0, \cdots, n$ is given by

$$
B_{i}(s)= \begin{cases}\frac{1}{\left(\tau_{i}-\tau_{i+1}\right)}\left(s-\tau_{i+1}\right) & s \in\left[\tau_{i+1}, \tau_{i}\right], \\ \frac{1}{\left(\tau_{i-1}-\tau_{i}\right)}\left(\tau_{i-1}-s\right) & s \in\left[\tau_{i}, \tau_{i-1}\right], \\ 0 & \text { otherwise. }\end{cases}
$$

Namely, $B_{i}(s), i=0, \cdots, n$ are piecewise linear functions. After substituting the special form of $\xi$ in Equation (16) into Equations (14)-(15), the governing equations for $\kappa_{i}(t), i=0, \cdots, n$ become the following:

$$
\begin{gathered}
\frac{\mathrm{d}}{\mathrm{d} t} \kappa_{i}(t)=\frac{1}{\delta_{i}}\left(\kappa_{i-1}(t)-\kappa_{i}(t)\right), i=1, \cdots, n, \\
\left\{\begin{array}{l}
\lambda \sum_{i=1}^{l} \frac{\mathrm{d}}{\mathrm{d} t} \kappa_{\bar{\sigma}_{i}}(t)+\lambda \int_{-1}^{0}|s|^{-p} \sum_{i=0}^{n} \kappa_{i}(t) \frac{\mathrm{d}}{\mathrm{d} s} B_{i}(s) \mathrm{d} s=u(t), \\
(1-\lambda) \int_{-1}^{0}|s|^{-p} \sum_{i=0}^{n} \kappa_{i}(t) \frac{\mathrm{d}}{\mathrm{d} s} B_{i}(s) \mathrm{d} s=v(t),
\end{array}\right.
\end{gathered}
$$

where $\delta_{i}=\tau_{i-1}-\tau_{i}>0$, for $i=1, \cdots, n$. For time $t$, discretization contains $T^{0}, T^{1}, \cdots, T^{m}$, for $0=T^{0}<T^{1}<\cdots<T^{m}=1$. Define $\Delta^{k}=T^{k+1}-T^{k}$, for $k=0, \cdots, m-1$. By assuming $\alpha_{i}^{k}=\kappa_{i}\left(T^{k}\right)$, for $i=0,1, \cdots, n$, and $k=0, \cdots, m$, and without losing generality, we assume $l=2, \bar{\sigma}_{1}=0, \bar{\sigma}_{2}=n$, and Equations (18)-(19) can now be written as

$$
\begin{gathered}
\frac{1}{\Delta^{k}}\left(\alpha_{i}^{k+1}-\alpha_{i}^{k}\right)=\frac{1}{\delta_{i}}\left(\alpha_{i-1}^{k}-\alpha_{i}^{k}\right), \\
\left\{\begin{array}{l}
\frac{\lambda}{\delta_{1}} \alpha_{0}^{k+1}-\frac{\lambda}{\delta_{1}} \alpha_{1}^{k+1}+\frac{\lambda}{\delta_{n-1}} \alpha_{n-1}^{k+1}-\frac{\lambda}{\delta_{n-1}} \alpha_{n}^{k+1}+\lambda \sum_{i=1}^{n} \frac{g_{i}}{\delta_{i}}\left(\alpha_{i-1}^{k+1}-\alpha_{i}^{k+1}\right)=u\left(T^{k+1}\right), \\
(1-\lambda) \sum_{i=1}^{n} \frac{g_{i}}{\delta_{i}}\left(\alpha_{i-1}^{k+1}-\alpha_{i}^{k+1}\right)=v\left(T^{k+1}\right),
\end{array}\right.
\end{gathered}
$$

for $i=1, \cdots, n, \quad k=0, \cdots, m-1$, and $g_{i}=\int_{\tau_{i}}^{\tau_{i-1}}|s|^{-p} \mathrm{~d} s$.

Furthermore, we assume a uniform mesh for both space and time, and the mesh points are $\tau_{i}, i=0, \cdots, n$ and $T^{k}, k=0, \cdots, m$. Specifically, we have $\tau_{i}=-\frac{i}{n}$, $T^{k}=\frac{k}{m}$, for some positive integers $n$ and $m$. The associated differences are defined as $\Delta^{k}=T^{k+1}-T^{k}, \quad k=0, \cdots, m-1$, for the time variable and $\delta_{i}=\tau_{i-1}-\tau_{i}$, $i=1, \cdots, n$, for the space variable. Thus, we obtain $\Delta^{k}=1 / m$ and $\delta_{i}=1 / n$, for $k=0, \cdots, m-1$, and $i=1, \cdots, n$. Setting $m=n$ produces the relation $\Delta^{k}=\delta_{i}=1 / n$ for $k=0, \cdots, n-1$, and $i=1, \cdots, n$, and deriving Equations (20)-(21) lead to the following system:

$$
\alpha_{i}^{k+1}=\alpha_{i-1}^{k},
$$


and

$$
\left\{\begin{array}{c}
\frac{\lambda}{\delta_{1}} \alpha_{0}^{k+1}-\frac{\lambda}{\delta_{1}} \alpha_{1}^{k+1}+\frac{\lambda}{\delta_{n-1}} \alpha_{n-1}^{k+1}-\frac{\lambda}{\delta_{n-1}} \alpha_{n}^{k+1}+\lambda \sum_{i=1}^{n} \frac{1}{\delta_{i}}\left(\alpha_{i-1}^{k+1}-\alpha_{i}^{k+1}\right) \cdot \frac{1}{1-p}\left[-\left(-\tau_{i-1}\right)^{1-p}+\left(-\tau_{i}\right)^{1-p}\right]=u\left(T^{k+1}\right)=u_{k+1} \\
(1-\lambda) \sum_{i=1}^{n} \frac{1}{\delta_{i}}\left(\alpha_{i-1}^{k+1}-\alpha_{i}^{k+1}\right) \cdot \frac{1}{1-p}\left[-\left(-\tau_{i-1}\right)^{1-p}+\left(-\tau_{i}\right)^{1-p}\right]=v\left(T^{k+1}\right) \equiv v_{k+1} \\
\text { for } i=1, \cdots, n, \text { and } k=0, \cdots, n-1 .
\end{array}\right.
$$

After defining corresponding constants $c_{0}, c_{1}, \cdots, c_{n}$, and $d_{0}, d_{1}, \cdots, d_{n}$, Equation (23) can be written in the following simplified form:

$$
\left\{\begin{array}{l}
\lambda\left(\alpha_{0}^{k+1} c_{0}+\alpha_{0}^{k} c_{1}+\cdots+\alpha_{0}^{0} c_{k+1}+\cdots+\alpha_{n-k-1}^{0} c_{n}\right)=u_{k+1} \\
(1-\lambda)\left(\alpha_{0}^{k+1} d_{0}+\alpha_{0}^{k} d_{1}+\cdots+\alpha_{0}^{0} d_{k+1}+\cdots+\alpha_{n-k-1}^{0} d_{n}\right)=v_{k+1}
\end{array} \quad, k=0, \cdots, n-1\right.
$$

The connection between the solution $x(t)$ and $\alpha$ 's is as follows: Because $\xi(t, s)=x(t+s)$, for $-1 \leq s \leq 0, t>0$, and $\xi(t, s)=\sum^{n} \kappa_{i}(t) B_{i}(s)$, it follows that $x(\bar{t})$, for $\bar{t}>0$ can be obtained in the following case:

$$
x\left(T^{j}\right)=\sum_{l=0}^{n} \kappa_{l}\left(T^{j}\right) B_{l}(0)=\kappa_{0}\left(T^{j}\right)=\alpha_{0}^{j}, \text { for } j=1, \cdots, n .
$$

For the cost function

$$
\Phi_{1}(\lambda)=\alpha_{1}(\lambda x(1)-h)^{2}+\alpha_{2} \int_{0}^{1}(\lambda x(t)-\eta(t))^{2} \mathrm{~d} t+\alpha_{3} \int_{0}^{1} u(t)^{2} \mathrm{~d} t
$$

the discretized form is:

$$
\Phi_{1}(\lambda)=\alpha_{1}\left(\lambda \alpha_{0}^{n}-h\right)^{2}+\alpha_{2} \frac{1}{n} \sum_{i=1}^{n}\left(\lambda \alpha_{0}^{i}-\eta\left(T^{i}\right)\right)^{2}+\alpha_{3} \frac{1}{n} \sum_{i=1}^{n} u_{i}^{2} .
$$

Taking the first derivatives of $\Phi_{1}(\lambda)$ with respect to $u_{i}, i=1, \cdots, n$, and setting them to zero yields the following equations:

$$
\begin{gathered}
n \lambda^{2} \alpha_{1} \cdot \alpha_{0}^{n} \cdot a a(n)+\lambda^{2} \alpha_{2}\left[a a(1) \cdot \alpha_{0}^{1}+a a(2) \cdot \alpha_{0}^{2}+\cdots+a a(n) \cdot \alpha_{0}^{n}\right]+\alpha_{3} \cdot u_{1} \\
=n \lambda \alpha_{1} h \cdot a a(n)+\lambda \alpha_{2}\left[\eta\left(t_{1}\right) \cdot a a(1)+\cdots+\eta\left(t_{n}\right) \cdot a a(n)\right] \\
\vdots \\
n \lambda^{2} \alpha_{1} \cdot \alpha_{0}^{n} \cdot a a(n-j+1)+\lambda^{2} \alpha_{2}\left[a a(1) \cdot \alpha_{0}^{j}+a a(2) \cdot \alpha_{0}^{j+1}+\cdots\right. \\
\left.+a a(n-j+1) \cdot \alpha_{0}^{n}\right]+\alpha_{3} \cdot u_{j} \\
=n \lambda \alpha_{1} h \cdot a a(n-j+1)+\lambda \alpha_{2}\left[\eta\left(t_{j}\right) \cdot a a(1)+\cdots+\eta\left(t_{n}\right) \cdot a a(n-j+1)\right] \\
\vdots \\
n \lambda^{2} \alpha_{1} \cdot \alpha_{0}^{n} \cdot a a(1)+\lambda^{2} \alpha_{2} \cdot \alpha_{0}^{n} \cdot a a(1)+\alpha_{3} \cdot u_{n} \\
=n \lambda \alpha_{1} h \cdot a a(1)+\lambda \alpha_{2} \cdot \eta\left(t_{n}\right) \cdot a a(1)
\end{gathered}
$$

where

$$
\begin{gathered}
a a(1)=\frac{1}{\lambda c_{0}}, \\
a a(2)=-\frac{c_{1}}{c_{0}} \cdot a a(1), \\
\vdots \\
a a(j)=-\frac{c_{1}}{c_{0}} a a(j-1)-\frac{c_{2}}{c_{0}} \cdot a a(j-2)-\cdots-\frac{c_{j-1}}{c_{0}} \cdot a a(1),
\end{gathered}
$$




$$
a a(n)=-\frac{c_{1}}{c_{0}} a a(n-1)-\frac{c_{2}}{c_{0}} \cdot a a(n-2)-\cdots-\frac{c_{n-1}}{c_{0}} \cdot a a(1) .
$$

Systems (24) with $\lambda$ and (27) can be set up as $[A][x]=[b]$, where the vector $[x]$ consists of the unknowns $\alpha_{0}^{j}, j=1, \cdots, n$, and $u_{k}, k=1, \cdots, n$. The structure of matrix $[A]$ is

$\left[\begin{array}{cccccccc}\lambda c_{0} & 0 & \cdots & 0 & -1 & 0 & \cdots & 0 \\ \lambda c_{1} & \lambda c_{0} & \cdots & 0 & 0 & -1 & \cdots & 0 \\ \vdots & \vdots & \ddots & \vdots & \vdots & \vdots & \ddots & \vdots \\ \lambda c_{n-1} & \lambda c_{n-2} & \cdots & c_{0} & 0 & 0 & \cdots & -1 \\ \lambda^{2} \alpha_{2} a a(1) & \lambda^{2} \alpha_{2} a a(2) & \cdots & \lambda^{2}\left(\alpha_{2}+n \alpha_{1}\right) a a(n) & \alpha_{3} & 0 & \cdots & 0 \\ 0 & \lambda^{2} \alpha_{2} a a(1) & \cdots & \vdots & 0 & \alpha_{3} & \cdots & 0 \\ \vdots & \vdots & \ddots & \lambda^{2}\left(\alpha_{2}+n \alpha_{1}\right) a a(3) & \vdots & \vdots & \ddots & \vdots \\ \vdots & \vdots & \lambda^{2} \alpha_{2} a a(1) & \lambda^{2}\left(\alpha_{2}+n \alpha_{1}\right) a a(2) & & \alpha_{3} & 0 \\ 0 & 0 & \cdots & \lambda^{2}\left(\alpha_{2}+n \alpha_{1}\right) a a(1) & 0 & \cdots & 0 & \alpha_{3}\end{array}\right]_{2 n \times 2 n}$

and vector $[b]$ is given by

$$
\lambda\left[\begin{array}{c}
-\alpha_{0}^{0} c_{1}-\alpha_{1}^{0} c_{2}-\cdots-\alpha_{n-1}^{0} c_{n}-b\left(t_{1}\right) \\
-\alpha_{0}^{0} c_{2}-\alpha_{1}^{0} c_{3}-\cdots-\alpha_{n-2}^{0} c_{n}-b\left(t_{2}\right) \\
-\alpha_{0}^{0} c_{3}-\alpha_{1}^{0} c_{4}-\cdots-\alpha_{n-3}^{0} c_{n}-b\left(t_{3}\right) \\
\vdots \\
-\alpha_{0}^{0} c_{n}-b\left(t_{n}\right) \\
\alpha_{2}\left[\eta\left(t_{1}\right) a a(1)+\cdots+\eta\left(t_{n-1}\right) a a(n-1)\right]+\left[\alpha_{2} \eta\left(t_{n}\right)+n \alpha_{1} h\right] a a(n) \\
\alpha_{2}\left[\eta\left(t_{2}\right) a a(1)+\cdots+\eta\left(t_{n-1}\right) a a(n-2)\right]+\left[\alpha_{2} \eta\left(t_{n}\right)+n \alpha_{1} h\right] a a(n-1) \\
\vdots \\
{\left[\alpha_{2} \eta\left(t_{n}\right)+n \alpha_{1} h\right] a a(1)}
\end{array}\right]_{2 n \times 1}
$$

For the cost function

$$
\Phi_{2}(\lambda)=\alpha_{1}((1-\lambda) x(1)-h)^{2}+\alpha_{2} \int_{0}^{1}((1-\lambda) x(t)-\eta(t))^{2} \mathrm{~d} t+\alpha_{3} \int_{0}^{1} v(t)^{2} \mathrm{~d} t,
$$

the discretized form is:

$$
\Phi_{2}(\lambda)=\alpha_{1}\left((1-\lambda) \alpha_{0}^{n}-h\right)^{2}+\alpha_{2} \frac{1}{n} \sum_{i=1}^{n}\left((1-\lambda) \alpha_{0}^{i}-\eta\left(T^{i}\right)\right)^{2}+\alpha_{3} \frac{1}{n} \sum_{i=1}^{n} v_{i}^{2} .
$$

Taking first derivatives of $\Phi_{2}(\lambda)$ with respect to $v_{i}, i=1, \cdots, n$, and setting them to zero produces the following equations:

$$
\begin{aligned}
& n(1-\lambda)^{2} \alpha_{1} \cdot \alpha_{0}^{n} \cdot a a(n)+(1-\lambda)^{2} \alpha_{2}\left[a a(1) \cdot \alpha_{0}^{1}+a a(2) \cdot \alpha_{0}^{2}+\cdots\right. \\
& \left.+a a(n) \cdot \alpha_{0}^{n}\right]+\alpha_{3} \cdot v_{1} \\
& =n(1-\lambda) \alpha_{1} h \cdot a a(n)+(1-\lambda) \alpha_{2}\left[\eta\left(t_{1}\right) \cdot a a(1)+\cdots+\eta\left(t_{n}\right) \cdot a a(n)\right], \\
& \quad \vdots \\
& n(1-\lambda)^{2} \alpha_{1} \cdot \alpha_{0}^{n} \cdot a a(n-j+1)+(1-\lambda)^{2} \alpha_{2}\left[a a(1) \cdot \alpha_{0}^{j}+a a(2) \cdot \alpha_{0}^{j+1}+\cdots\right. \\
& \left.+a a(n-j+1) \cdot \alpha_{0}^{n}\right]+\alpha_{3} \cdot v_{j} \\
& =n(1-\lambda) \alpha_{1} h \cdot a a(n-j+1)+\lambda \alpha_{2}\left[\eta\left(t_{j}\right) \cdot a a(1)+\cdots+\eta\left(t_{n}\right) \cdot a a(n-j+1)\right],
\end{aligned}
$$




$$
\begin{aligned}
& (1-\lambda)^{2} \alpha_{0}^{n}\left(n \cdot \alpha_{1} \cdot a a(1)\right)+\alpha_{2} \cdot a a(1)+\alpha_{3} \cdot v_{n} \\
& =(1-\lambda) a a(1)\left[n \alpha_{1} h+\alpha_{2} \cdot \eta\left(t_{n}\right)\right] .
\end{aligned}
$$

where

$$
\begin{gathered}
a a(1)=\frac{1}{(1-\lambda) c_{0}}, \\
a a(2)=-\frac{c_{1}}{c_{0}} \cdot a a(1), \\
\vdots \\
a a(j)=-\frac{c_{1}}{c_{0}} a a(j-1)-\frac{c_{2}}{c_{0}} \cdot a a(j-2)-\cdots-\frac{c_{j-1}}{c_{0}} \cdot a a(1), \\
\vdots \\
a a(n)=-\frac{c_{1}}{c_{0}} a a(n-1)-\frac{c_{2}}{c_{0}} \cdot a a(n-2)-\cdots-\frac{c_{n-1}}{c_{0}} \cdot a a(1) .
\end{gathered}
$$

Systems (24) with $1-\lambda$ and (29) can be set up as $[A][x]=[b]$, where the vector $[x]$ consists of the unknowns $\alpha_{0}^{j}, j=1, \cdots, n$, and $v_{k}, k=1, \cdots, n$. The structure of matrix $[A]$ is

$\left[\begin{array}{cccccccc}(1-\lambda) d_{0} & 0 & \cdots & 0 & -1 & 0 & \cdots & 0 \\ (1-\lambda) d_{1} & (1-\lambda) d_{0} & \cdots & \ddots & 0 & -1 & \cdots & 0 \\ \vdots & \vdots & \ddots & \vdots & \vdots & \ddots & \vdots \\ (1-\lambda) d_{n-1} & (1-\lambda) d_{n-2} & \cdots & d_{0} & 0 & 0 & \cdots & -1 \\ (1-\lambda)^{2} \alpha_{2} a a(1) & (1-\lambda)^{2} \alpha_{2} a a(2) & \cdots & (1-\lambda)^{2}\left(\alpha_{2}+n \alpha_{1}\right) a a(n) & \alpha_{3} & 0 & \cdots & 0 \\ 0 & (1-\lambda)^{2} \alpha_{2} a a(1) & \cdots & 0 & \alpha_{3} & \cdots & 0 \\ \vdots & \vdots & \ddots & (1-\lambda)^{2}\left(\alpha_{2}+n \alpha_{1}\right) a a(3) & \vdots & \vdots & \ddots & \vdots \\ \vdots & \vdots & (1-\lambda)^{2} \alpha_{2} a a(1) & (1-\lambda)^{2}\left(\alpha_{2}+n \alpha_{1}\right) a a(2) & & \alpha_{3} & 0 \\ 0 & 0 & \cdots & (1-\lambda)^{2}\left(\alpha_{2}+n \alpha_{1}\right) a a(1) & 0 & \cdots & 0 & \alpha_{3}\end{array}\right]_{2 n \times 2 n}$

and vector $[b]$ is given by

$$
(1-\lambda)\left[\begin{array}{c}
-\alpha_{0}^{0} d_{1}-\alpha_{1}^{0} d_{2}-\cdots-\alpha_{n-1}^{0} d_{n}-b\left(t_{1}\right) \\
-\alpha_{0}^{0} d_{2}-\alpha_{1}^{0} d_{3}-\cdots-\alpha_{n-2}^{0} d_{n}-b\left(t_{2}\right) \\
-\alpha_{0}^{0} d_{3}-\alpha_{1}^{0} d_{4}-\cdots-\alpha_{n-3}^{0} d_{n}-b\left(t_{3}\right) \\
\vdots \\
-\alpha_{0}^{0} d_{n}-b\left(t_{n}\right) \\
\alpha_{2}\left[\eta\left(t_{1}\right) a a(1)+\cdots+\eta\left(t_{n-1}\right) a a(n-1)\right]+\left[\alpha_{2} \eta\left(t_{n}\right)+n \alpha_{1} h\right] a a(n) \\
\alpha_{2}\left[\eta\left(t_{2}\right) a a(1)+\cdots+\eta\left(t_{n-1}\right) a a(n-2)\right]+\left[\alpha_{2} \eta\left(t_{n}\right)+n \alpha_{1} h\right] a a(n-1) \\
\vdots \\
{\left[\alpha_{2} \eta\left(t_{n}\right)+n \alpha_{1} h\right] a a(1)}
\end{array}\right]_{2 n \times 1}
$$




\section{Numerical Examples}

Consider examples involving $p=0.5, \lambda \in[0,1]$, initial conditions $\phi(s)=0,-1 \leq s \leq 0$, different target final state $h$, and different target functions $\eta(t), 0 \leq t \leq 1$. For different criteria, the combinations of constants $\alpha$ s in the cost functions are changed accordingly.

For the case $\left(\alpha_{1}, \alpha_{2}, \alpha_{3}\right)=(0,1,0)$, the problem is the "tracking problem".

Typical cost distribution is as the following two graphs (Figure 1 and Figure 2).

Example 1: $n=100,\left(\alpha_{1}, \alpha_{2}, \alpha_{3}\right)=(0.3,0.5,0.2)$

\begin{tabular}{cccc}
\hline$h=1$ & $\eta(t)=1$ & mincost $\Phi=0.5951$ & when $\lambda=0$ \\
$h=1$ & $\eta(t)=t$ & mincost $\Phi=0.3313$ & when $\lambda=0$ \\
$h=0$ & $\eta(t)=1-t$ & mincost $\Phi=0.1517$ & when $\lambda=0$ \\
\hline
\end{tabular}

Example 2: $n=100,\left(\alpha_{1}, \alpha_{2}, \alpha_{3}\right)=(0,0,1)$

\begin{tabular}{llll}
\hline$h=1$ & $\eta(t)=1$ & mincost $\Phi=0$ & when $\lambda=0$ \\
$h=1$ & $\eta(t)=t$ & mincost $\Phi=0$ & when $\lambda=0$ \\
$h=0$ & $\eta(t)=1-t$ & mincost $\Phi=0$ & when $\lambda=0$ \\
\hline
\end{tabular}

Example 3: $n=100,\left(\alpha_{1}, \alpha_{2}, \alpha_{3}\right)=(1,0,0)$

\begin{tabular}{cccc}
\hline$h=1$ & $\eta(t)=1$ & mincost $\Phi=2.2132 e-28$ & when $\lambda=0$ \\
$h=1$ & $\eta(t)=t$ & mincost $\Phi=2.2132 e-28$ & when $\lambda=0$ \\
$h=0$ & $\eta(t)=1-t$ & mincost $\Phi=0$ & when $\lambda=0$ \\
\hline
\end{tabular}

Example 4: $n=100,\left(\alpha_{1}, \alpha_{2}, \alpha_{3}\right)=(0,1,0)$

\begin{tabular}{cccc}
\hline$h=1$ & $\eta(t)=1$ & mincost $\Phi=5.8587 e-29$ & when $\lambda=0$ \\
$h=1$ & $\eta(t)=t$ & mincost $\Phi=2.5009 e-29$ & when $\lambda=0$ \\
$h=0$ & $\eta(t)=1-t$ & mincost $\Phi=2.9966 e-29$ & when $\lambda=0$ \\
\hline
\end{tabular}

Example 5: $n=100,\left(\alpha_{1}, \alpha_{2}, \alpha_{3}\right)=(0.9,0,0.1)$

\begin{tabular}{cccc}
\hline$h=1$ & $\eta(t)=1$ & mincost $\Phi=0.3424$ & when $\lambda=0$ \\
$h=1$ & $\eta(t)=t$ & mincost $\Phi=0.3424$ & when $\lambda=0$ \\
$h=0$ & $\eta(t)=1-t$ & mincost $\Phi=0$ & when $\lambda=0$ \\
\hline Example 6: $h=100,\left(\alpha_{1}, \alpha_{2}, \alpha_{3}\right)=(0,0.9,0.1)$ & & \\
\hline$h=1$ & $\eta(t)=1$ & mincost $\Phi=0.5712$ & when $\lambda=0$ \\
\hline$h=1$ & mincost $\Phi=0.1785$ & when $\lambda=0.5$ \\
$h=0$ & mincost $\Phi=0.2321$ & when $\lambda=0$ \\
\hline
\end{tabular}




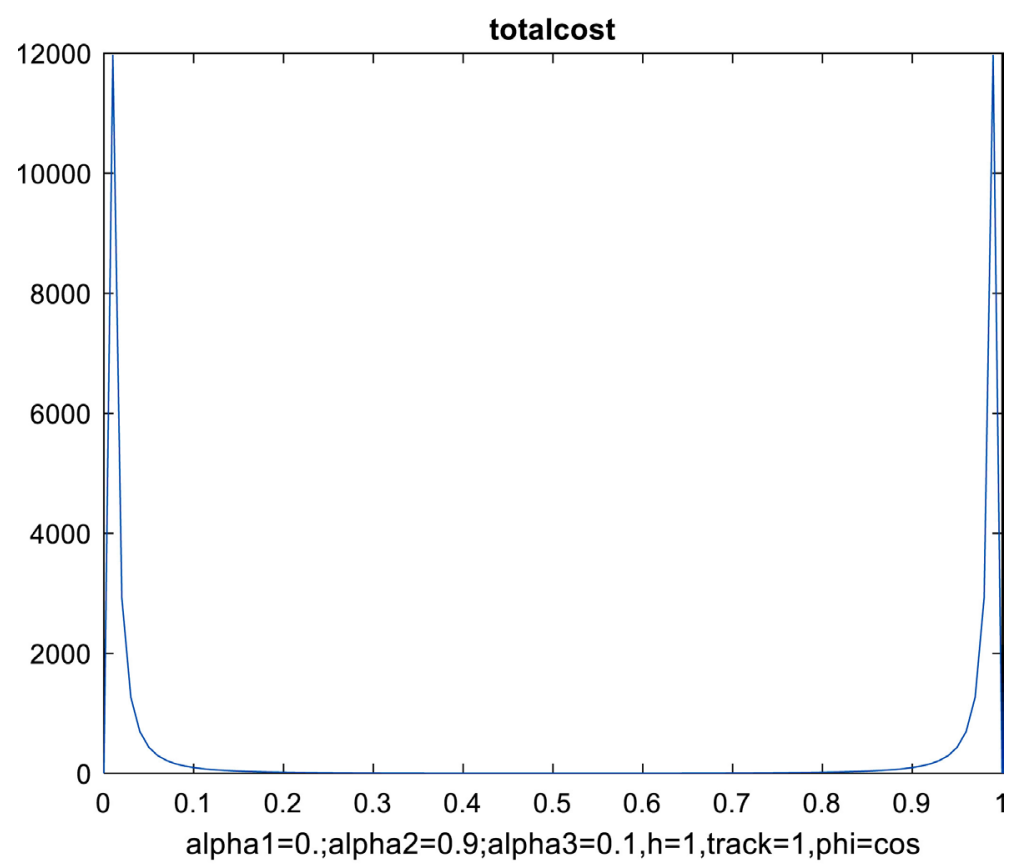

Figure 1. Total cost for $\lambda$ from 0 to 1 .

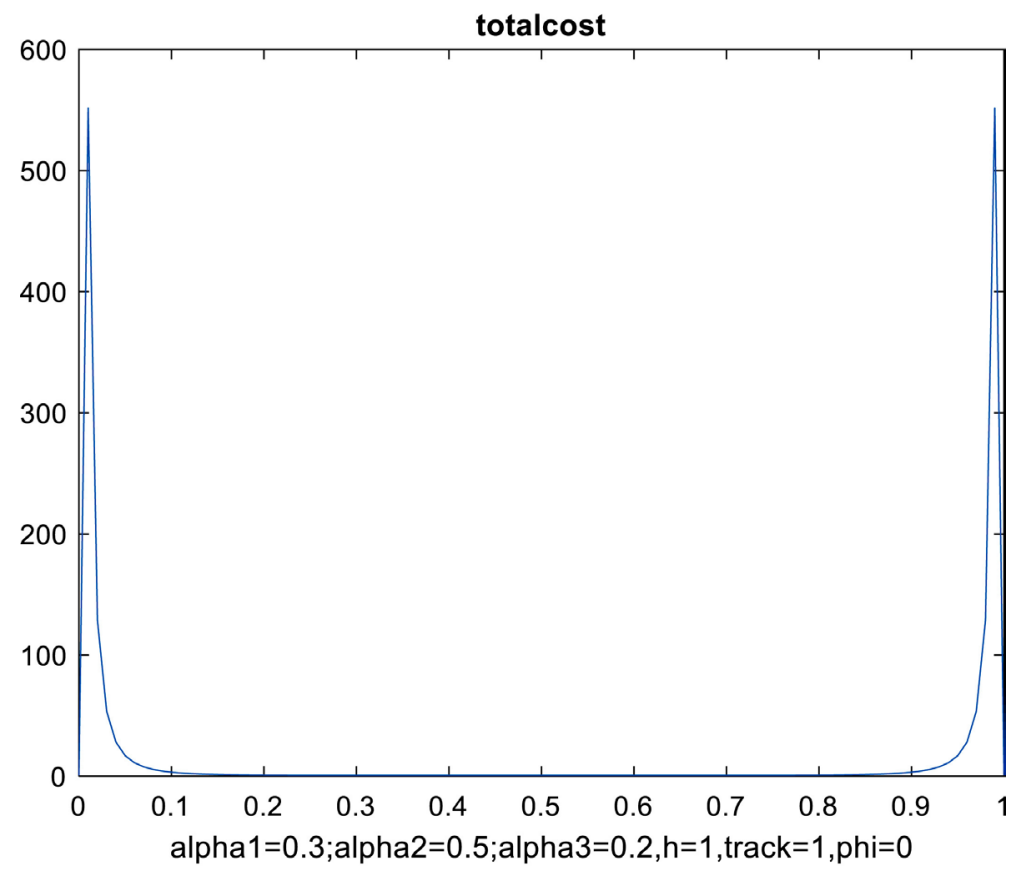

Figure 2. Total cost for $\lambda$ from 0 to 1 .

\section{Conclusion}

This study presented a numerical method for finding the minimum of the total cost when it contains two partial costs from two dynamic systems, and each cost contains three weights to adjust for different considerations of energy and different combinations of the measurable parameter $\lambda$ between two systems. The effectiveness of the proposed method was tested by examples. The numerical re- 
sults indicated that the most stable situations are $\lambda=0$. In other words, dynamic system with the first kind integro-differential equation is the most stable system in the minimum cost sense.

\section{Acknowledgements}

Author would like to thank MOST (Ministry of Science and Technology) under grant No.108-2914-I-216-002-A1 to partially support this project.

\section{Conflicts of Interest}

The author declares no conflicts of interest regarding the publication of this paper.

\section{References}

[1] Burns, J.A., Cliff, E.M. and Herdman, T.L. (1983) A State-Space Model for an Aeroelastic System. Proceedings of 22nd IEEE Conference on Decision and Control, San Antonio, December 1983, 1074-1077. https://doi.org/10.1109/CDC.1983.269685

[2] Burns, J.A., Herdman, T.L. and Stech, H.W. (1983) Linear Functional Differential Equations as Semigroups on Product Spaces. SIAM Journal on Mathematical Analysis, 14, 98-116. https://doi.org/10.1137/0514007

[3] Kappel, F. and Zhang, K.P. (1986) Equivalence of Functional Equations of Neutral Type and Abstract Cauchy Problems. Monatshefte für Mathematik, 101, 115-133. https://doi.org/10.1007/BF01298925

[4] Vrbka, L. and Jungwirth, P. (2006) Homogeneous Freezing of Water Starts in the Subsurface. The Journal of Physical Chemistry B, 110, 18126-18129. https://doi.org/10.1021/jp064021c

[5] Vorsin, D. and Arogeti, S. (2017) Flight Transition Control of a Multipurpose UAV. 201713 th IEEE International Conference on Control \& Automation (ICCA), Ohrid, 3-6 July 2017, 507-512. https://doi.org/10.1109/ICCA.2017.8003112

[6] Chiang, S.C. and Herdman, T.L. (2013) Revised Numerical Methods for Optimal Control of a Class of Singular Integro-Differential Equations. Mathematics in Engineering, Science and Aerospace, 4, 171-187. 